\title{
A novel mechanism for parallel conduction in GaAs-(Ga,Al)As heterojunctions
}

\author{
R M Kusterst§, T J B M Janssent, C J G M Langerak† $\|$, \\ J SingletontI, J A A J Perenboomt, G A C Jonesł, \\ D A Ritchieł and J E F Frostł
}

† High Field Magnet Laboratory and Research Institute for Materials, University of Nijmegen, Toernooiveld, NL-6525 ED Nijmegen, The Netherlands $¥$ Cavendish Laboratory, University of Cambridge, Madingley Road, Cambridge CB3 OHE, UK

Received 14 October 1991, in final form 25 February 1992, accepted for publication 18 March 1992

\begin{abstract}
GaAs-(Ga,Al)As heterojunctions often show parallel conduction after illumination with red light $\left(\hbar \omega>E_{g}\right)$. A study of the parallel conduction occurring in planar doped GaAs-(Ga,Al)As heterojunctions with a superlattice buffer is presented. The samples studied are subject to parallel conduction by two different types of carrier. Transport measurements indicate the presence of a very large density of low-mobility carriers, while in addition the presence of some high-mobility carriers is indicated by the appearance of an additional dip in the far-infrared (FIR) transmission. The latter type of carrier is thought to form a secondary low-density two-dimensional electron gas weakly confined behind the superlattice buffer.
\end{abstract}

\section{Introduction}

One of the most attractive properties of modulationdoped $\mathrm{GaAs}-(\mathrm{Ga}, \mathrm{Al}) \mathrm{As}$ heterojunctions is the very high electron mobility achieved in these structures, which can be exploited in transistors with very high switching speed. However, the performance of such a transistor (high electron mobility transistor, HEMT) is considerably reduced if there are more conducting paths than through the active layer of the two-dimensional electron gas (2DEG) [1]. The most probable cause of parallel conduction in GaAs- $(\mathrm{Ga}, \mathrm{Al}) \mathrm{As}$ heterojunctions is the presence of conduction band $(\mathrm{CB})$ electrons in the $(\mathrm{Ga}, \mathrm{Al}) \mathrm{As}$ layer [1-3], and this depends on the doping parameters (doping concentration, spacer width, thickness of the (Ga,Al)As layer) and $\mathrm{Al}$ content of this layer [1]. The latter determines the $\mathrm{CB}$ potential discontinuity at the interface: it must be at least $\approx 0.3$ to prevent parallel conduction. The discontinuity in the

$\S$ Present address: Royal Dutch/Shell Exploration and Production Laboratory, NL-2280 AB Rijswijk, The Netherlands.

|| Present address: University of Nottingham, Nottingham NG7 2RD, UK.

I Present address: Clarendon Laboratory, University of Oxford, Oxford OX1 3PU, UK. potential at the interface must be at least some tens of meV higher than the product of the spacer width and the interface electric field, which is proportional to the sum of the 2D carrier density and the depletion charge density. Parallel conduction occurs if there is a region in the $(\mathrm{Ga}, \mathrm{Al}) \mathrm{As}$ where the $\mathrm{CB}$ edge is lower than the Fermi energy $E_{\mathrm{F}}$. Another important parameter determining the presence of parallel conduction is the density of ionized donors $n_{\mathrm{D}}(z)$, which is proportional to the second spatial derivative $\mathrm{d} V_{\mathrm{CB}}^{2} / \mathrm{d} z^{2}$ of the CB-edge potential ( $z$ is the direction perpendicular to the $2 \mathrm{DEG})$. In our samples $n_{\mathrm{D}}(z)$ has a very high and narrow peak at the position of the planar Si doping.

Parallel conduction can be a result of the persistent photoconductivity (PPC) effect [4], which leads to ionization of deep donor states (DX centres [5]), thus increasing the effective value of $n_{\mathrm{D}}(z)$, which changes $V_{\mathrm{CB}}(z)$ in the $(\mathrm{Ga}, \mathrm{Al}) \mathrm{As}$ and the electric field at the interface.

We have observed such an onset of parallel conduction in three MBE-grown heterojunctions after a certain amount of illumination with red light. These samples are different from conventional $\mathrm{GaAs}-(\mathrm{Ga}, \mathrm{Al}) \mathrm{As}$ heterojunctions: the $(\mathrm{Ga}, \mathrm{Al})$ As layer is undoped except for a sheet of $5 \times 10^{16} \mathrm{~m}^{-2} \mathrm{Si}$ donors at a distance $d$ from the interface; $d$ is 5,10 and $20 \mathrm{~nm}$ respectively in the 


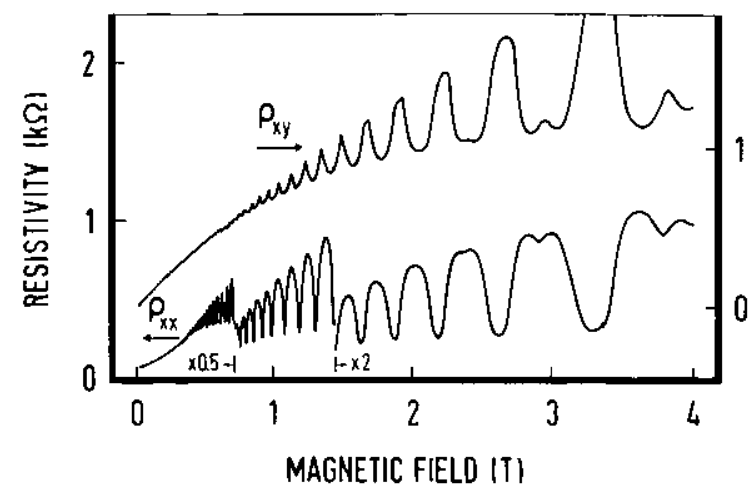

Figure 1. Typical traces of the diagonal and Hall resistivity observed in sample 3 after saturation of the carrier density. Due to strong parallel conduction, the Hall resistance $\rho_{x y}$ is now dominated by $\mathrm{SdH}$ oscillations.

three different samples [6]. Furthermore, the samples have a $20 \times(2.5 \mathrm{~nm} \mathrm{GaAs}+2.5 \mathrm{~nm} \mathrm{AlAs})$ superlattice buffer to prevent dopant migration from the substrate [7].

The paper is divided into two parts. Section 2 deals with the transport data from the three samples after strong illumination. The results are discussed in terms of conduction by two different types of carrier: the electrons in the 2DEG and the parallel-conducting electrons. Sections 3 and 4 treat the observation of an additional peak in the far-infrared (FIR) absorption of two of our samples; this peak appears after strong illumination. An explanation for this phenomenon in terms of parallel conduction by weakly confined electrons in a GaAs layer is given: we assume that the growth scheme is responsible for the presence of the weakly confined parallel-conducting electrons.

\section{Magnetotransport: results and discussion}

Parallel conduction in high-mobility heterojunctions is usually observed in magnetotransport in that the highfield Shubnikov-de Haas $(\mathrm{SdH})$ minima no longer approach zero. Furthermore, the quantized Hall plateaux, usually observed in such high-mobility 2DEGs, are distorted. Figure 1 shows a set of $\rho_{x x}(B)$ and $\rho_{x y}(B)$ traces from sample 3 with a very large amount of parallel conduction. The behaviour is characterized by the fact that the minimum of the $\mathrm{SdH}$ envelope shows a quadratic magnetoresistance at relatively low ficlds, is approximately linear at intermediate fields, and tends to saturate at fields well above $20 \mathrm{~T}$. As long as the parallel conduction is weak the distortion shows up as a dip at the high-field side of the Hall plateaux (without parallel conduction the Hall resistance increases monotonically as a function of field). In the casc of strong parallel conduction (figure 1) the distortions have evolved into SdH-like oscillations with a sign opposite to the $\mathrm{SdH}$ oscillations in $\rho_{x x}$, and the Hall plateaux are so strongly distorted that they can no longer be recognized. This phenomenon can be explained by a mixing of $\rho_{x x}$ and $\rho_{x y}$ as a result of the short-circuiting of the voltages across the 2DEG by the parallcl-conducting layer.
Table 1. Estimated densities (in $\mathrm{m}^{-2}$ ) and mobilities (in $\mathrm{m}^{2} \mathrm{~V}^{-1} \mathrm{~s}^{-1}$ ) of the $2 \mathrm{DEG}$ and the parallel-conducting carriers after saturation of the persistent photoconductivity effect.

\begin{tabular}{lllll}
\hline Sample & $N_{\mathrm{s}}$ & $\left\langle\mu_{\text {2DEG }}\right\rangle$ & $N_{\| \text {cond }}$ & $\left\langle\mu_{\| \text {cond }}\right\rangle$ \\
\hline 1 & $16.3 \times 10^{15}$ & 8.5 & $\approx 50 \times 10^{15}$ & $\approx 0.07$ \\
2 & $11.1 \times 10^{15}$ & 52 & $\approx 50 \times 10^{15}$ & $\approx 0.08$ \\
3 & $6.57 \times 10^{15}$ & 74 & $\approx 50 \times 10^{15}$ & $\approx 0.09$ \\
\hline
\end{tabular}

The density $N_{\mathrm{s}}$ of the electrons in the 2DEG in each sample after saturation of the persistent photoconductivity was obtained from the period of the $\mathrm{SdH}$ oscillations; the results, accurate to $\pm 1 \%$, are shown in table 1. Approximate values of the 2DEG mobility $\left\langle\mu_{2 \mathrm{DEG}}\right\rangle$, and the mobility $\mu_{\| \text {cond }}$ and density $N_{\| \text {cond }}$ of the parallel-conducting carriers were deduced by applying the model of Kane et al [3]. The conditions required for the Kane model $\rho_{x y}=B /\left(N_{\mathrm{s}}+N_{\| \text {ond }}\right) e$ $\left(\rho_{x x}^{2} \ll \rho_{x y}^{2}\right.$ and $\mu_{\| \text {cond }} B \gg 1$ ) are only approximately satisfied, cven at our maximum field of $20 \mathrm{~T}$. $N_{\| \text {ond }}$ can therefore only be obtained approximately as its determination depends on a qualitative extrapolation of the data to higher fields. The values of the mobility given in table 1 also depend on the aspect ratios of the samples, and these could only be estimated $(\approx 2$ for sample 1 and $\approx 8 / 3$ for samples 2 and 3 ).

The results in table 1 show that saturation of the persistent photoconductivity effect gives rise to a very large number of parallel-conducting carriers with a low mobility. The most probable origin of the majority of these carriers is the $\delta$-dioping layer with $N_{\mathrm{Si}}=5 \times$ $10^{16} \mathrm{~m}^{-2}$; the carriers are expected to reside in the neighbourhood of this layer so that they are scattered very strongly by the ionized $\mathrm{Si}$ donors, leading to the low mobility.

In magnetotransport it is difficult to distinguish the presence of more than one parallel-conduction channel. In a qualitative way, however, their presence can be elucidated by combining the transport results with far-infrared (FIR) transmission data: different types of carrier may have different effective masses, and several different cyclotron resonances will then be observed.

\section{An additional far-infrared absorption peak}

We have performed FIR transmission spectroscopy on samples 1, 2 and 3 [6]. An optically pumped moleculargas FIR laser [8] has been used as a fixed-frequency source and the FIR transmission has been recorded as a function of field at $\approx 25$ different laser energies. Carbon bolometers were used as detector.

In the samples used in this study the total number of electrons in the 2DEG can be increased so much that the Fermi cnergy is above the band edge of the first excited electric subband of the confining well potential, and more than one subband will then be occupied. In figure 2 the FIR transmission of sample 2 after saturation 


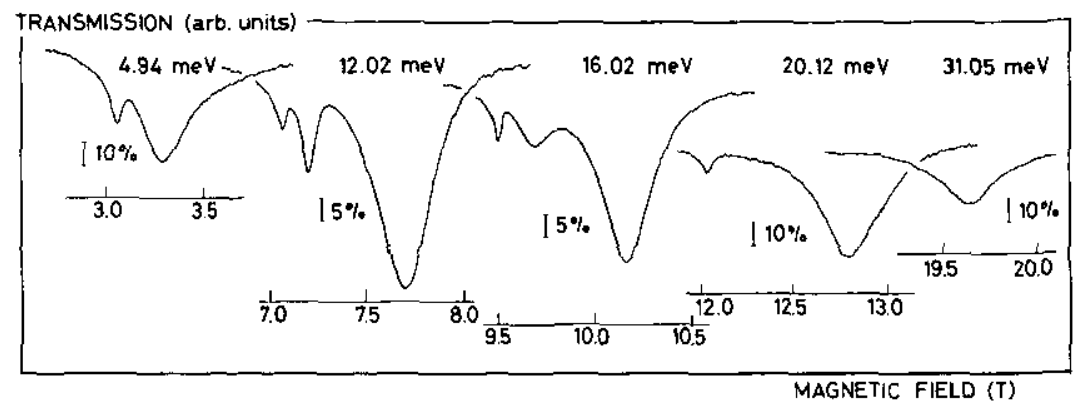

Figure 2. FIR transmission of sample 2 after saturation of the persistent photoconductivity effect at five different laser energies. Note the appearance of an additional resonance at $E_{\mathrm{FIR}}=12.02 \mathrm{meV}$.

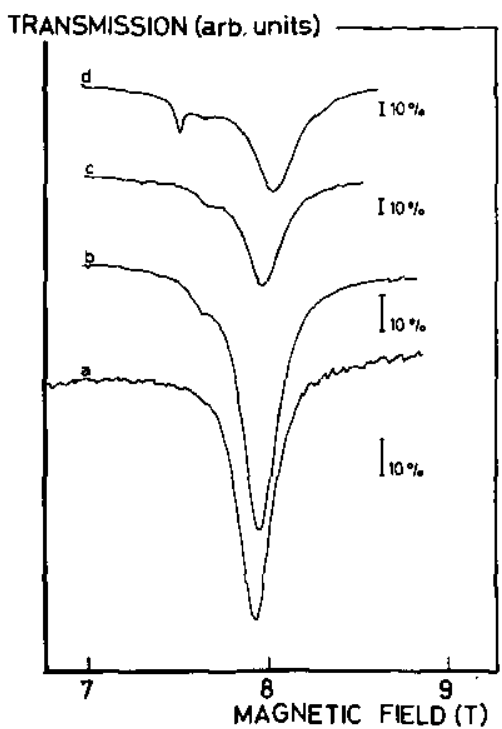

Figure 3. Evolution of the subband CRS and the $T_{3}$ resonance with successive increases of the $20 E G$ carrier density by bursts of red light; sample $2, E_{F I R}=12.85 \mathrm{meV}$.

of the carrier density is shown at five different laser energies. At an energy $E_{\mathrm{FIR}}=4.94 \mathrm{meV}$ we observe two dips due to $\mathrm{CR}$ : these originate from the carriers in the lowest and first-excited subband, and will be denoted as $\mathrm{CR}_{0}$ and $\mathrm{CR}_{1}$ respectively. At the $12.02 \mathrm{meV}$ laser line, however, a third resonance, which will be denoted as $T_{3}$, is seen at the low-field side of the subband CRs. This resonance grows in magnitude with increasing FIR energy. (Superposed on this general trend we see some fluctuations perhaps due to filling factor effects.) The growth of the $T_{3}$ resonance seems to coincide with a decrease of $\mathrm{CR}_{1}$. At still higher FIR energies (the trace taken at the $20.12 \mathrm{meV}$ line) we see that $\mathrm{CR}_{1}$ has disappeared. The $T_{3}$ resonance survives, but it decreases in amplitude and increases in linewidth. At the highest FIR energy we see only $\mathrm{CR}_{0}$.

Further FIR magnetotransmission measurements were performed in order to investigate the evolution of the different resonances with increasing carrier density. The carrier density was changed by controlled pulses of a red LED, and after each light pulse FIR transmission curves were taken at the $12.85 \mathrm{meV}$ and $16.02 \mathrm{meV}$

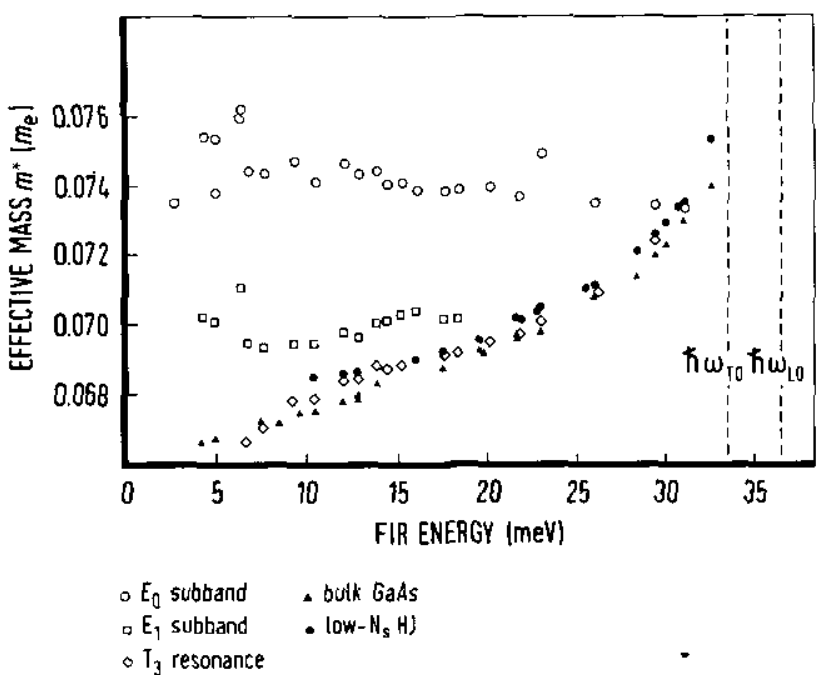

Figure 4. Effective masses extracted from the positions of the $T_{3}$ resonance as a function of FiR laser energy, and the subband effective masses $m_{0}^{*}$ and $m_{1}^{*}$. Cyclotron masses of low-density heterojunctions [9] and bulk GaAs cyclotron masses $[11,12]$ are given for comparison; sample $2_{1}$ $N_{\mathrm{s}}=1.16 \times 10^{16} \mathrm{~m}^{-2}$.

laser lines. The magnetoresistance was also measured to obtain the relevant subband carrier densities. Figure 3 shows a number of transmission traces taken at the $12.85 \mathrm{meV}$ line. Initially (curve a) a single $\mathrm{CR}$ is observed and no second periodicity was observed in the magnetoresistance. The next traces show the appearance of a second $\mathrm{CR}$ dip, and this is accompanied by the appearance of a second periodicity in the $\mathrm{SdH}$ oscillations. This indicates clearly that the second CR dip originates from the second-subband carriers. After a certain dose of light the onset of parallel conduction was observed. The very sharp $T_{3}$ resonance, however, emerged only after a few extra LED bursts, when the parallel conduction had a considerable strength. This suggests some relation between the $T_{3}$ resonance and parallel conduction.

From the position of the $T_{3}$ resonance as a function of laser energy the effective mass has been extracted. This effective mass is shown in figure 4 , together with the effective masses of the subband carriers. It shows a behaviour that is very similar to the cyclotron mass of elec- 

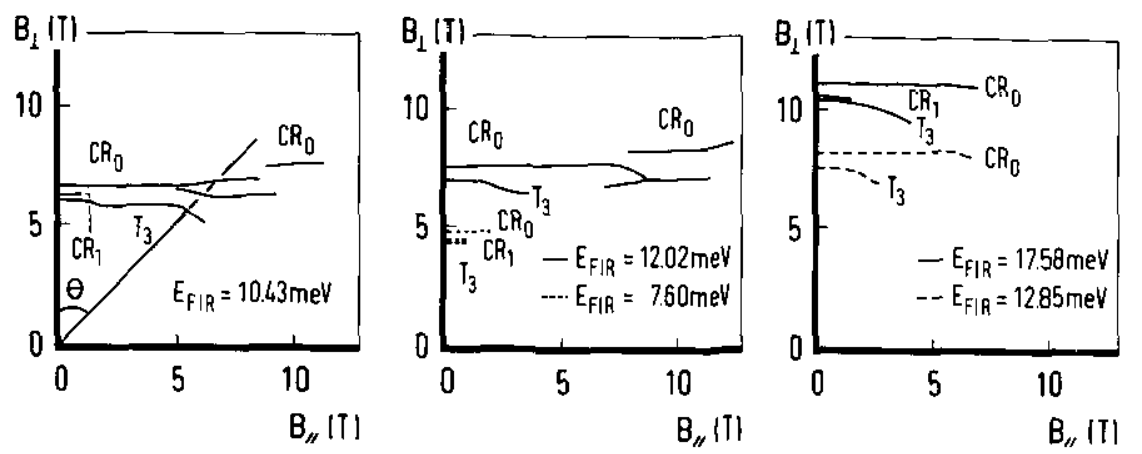

Figure 5. Polar diagrams of the resonance field of the subband CRS and the additional resonance $T_{3}$ as a function of tilt angle at several different laser energies: $7.60,10.43,12.02,12.85$ and 17.58 meV respectively; sample 2 , $N_{\mathrm{s}}=1.16 \times 10^{16} \mathrm{~m}^{-2}$. The tilt angle $\theta$ is shown in the leftmost diagram; the total applied field at resonance is given by $B^{2}=B_{\perp}^{2}+B_{\|}^{2} ; 20$ behaviour is characterized by $B_{\perp}=$ constant.

trons in low-carrier-density heterojunctions $[9,10]$ and bulk GaAs [11,12]. At energies approaching the energy of the longitudinal optic phonon $\hbar \omega_{L O}$ it shows a strong increase due to the resonant polaron effect $[9,10]$. The second-subband effective mass $m_{1}^{*}$ is lower than the lowest-subband effective mass $m_{0}^{*}$ due to GaAs conduction band non-parabolicity [9]. Band non-parabolicity is also responsible for the approximately linear increase with FIR energy well below $\hbar \omega_{\text {LO }}$ of all effective mass values except $m_{0}^{*}$; the latter is approximately constant due to the high density of the lowest-subband carriers [9]. Another effect of the high density of the lowestsubband carriers is the suppression of the resonant polaron effect: the mass does not significantly increase at FIR energies approaching $\hbar \omega_{\text {LO }}$. This suppression is due to screening and Landau level occupancy effects [9].

From the $\mathrm{SdH}$ spectra the carrier densities have been extracted, and the effective masses and CR linewidths have been obtained from the transmission curves. The two subband effective masses $m_{0}^{*}$ and $m_{1}^{*}$ tend to increase with total $2 \mathrm{DEG}$ sheet carricr density $N_{\mathrm{s}}$, but beyond a density of $\approx 8.4 \times 10^{15} \mathrm{~m}^{-2}$ the second-subband mass levels off; at this density also the $T_{3}$ resonance begins to appear. The lowest subband $C R$ linewidth shows an approximately linear increase with $N_{\mathrm{s}}$. This is consistent with the dominance of dielectric broadening of the $\mathrm{CR}[9,10]$.

The position of the $T_{3}$ resonance as a function of tilt angle is shown together with the positions of the subband CRs in figure 5. The tilt angle $\theta$ is indicated in the leftmost diagram: the applied magnetic field is given by $B=\sqrt{B_{\perp}^{2}+B_{\|}^{2}}$. The positions at which cyclotron resonances were found for $\approx 20$ different tilt angles have been plotted in the $B_{\perp} / B_{\|}$plane and connected with smooth curves. In these polar plots $2 \mathrm{D}$ behaviour will be indicated by straight lines $B_{\perp}=$ constant, and 3D behaviour by circles $B=$ constant. Three types of resonances were so identified: $\mathrm{CR}_{0}, \mathrm{CR}_{1}$ and $\mathrm{T}_{3}$. The position of the $T_{3}$ resonance shows $2 \mathrm{D}$ behaviour at $E_{\mathrm{FIR}}=7.60 \mathrm{meV}$. At higher laser energies it displays
3D behaviour at small tilt angles, but beyond a certain angle the behaviour changes into $2 \mathrm{D}$. The angle at which the crossover occurs increases with increasing laser energy. Not far beyond this angle the $\mathrm{T}_{3}$ resonance disappears, except at the $E_{\mathrm{FIR}}=10.43 \mathrm{meV}$ line. At the highest energy ( $E_{\mathrm{FIR}}=17.58 \mathrm{meV}$ ) the behaviour is 3D. The kind of behaviour described above has also been observed by Huant $e t$ al [13] in low-electron-density $\mathrm{GaAs}-(\mathrm{Ga}, \mathrm{Al})$ As heterojunctions; it has been attributed to subband-Landau-level coupling.

The resonances $\mathrm{CR}_{0}$ and $\mathrm{CR}_{1}$ occur at higher values of the perpendicular field component due to the higher effective masses. $\mathrm{CR}_{0}$ shows $2 \mathrm{D}$ bchaviour with anticrossings due to higher-order resonant subbandLandau-level coupling [14]. $\mathrm{CR}_{1}$ is only resolved at very small angles; it shows $2 \mathrm{D}$ behaviour at $E_{\mathrm{FIR}}=$ $7.6 \mathrm{meV}$ and $3 \mathrm{D}$ behaviour at $E_{\mathrm{FIR}}=17.58 \mathrm{meV}$. This is due to the large spatial extent of the second-subband envelope wavefunction. The mentioned behaviour also indicates that the separation $E_{21}$ between the third and the second subbands is in the range between the two values. This is in agreement with calculations by Ando [15] for a low depletion charge density.

\section{Discussion of the origin of the $T_{3}$ resonance}

In this section we will discuss a number of possible origins of the $T_{3}$ resonance. The growth scheme of the samples must be considered to determine which layers in the samples can give rise to this resonance. Figure 6 shows the growth scheme of samples 1,2 and 3: in these structures all materials are undoped except the GaAs cap layer and the Si planar doping in the $\mathrm{Ga}_{0.67} \mathrm{Al}_{0.33} \mathrm{As}$ containing the host donors of the 2DEG. As a result we get a high-density sheet of ionized donors at a distance of 5,10 or $20 \mathrm{~nm}$ from the interface respectively for the samples 1,2 and 3 [6]. We must now consider which of the layers of the structure of figure 6 (top) can give rise to the $T_{3}$ resonance. 

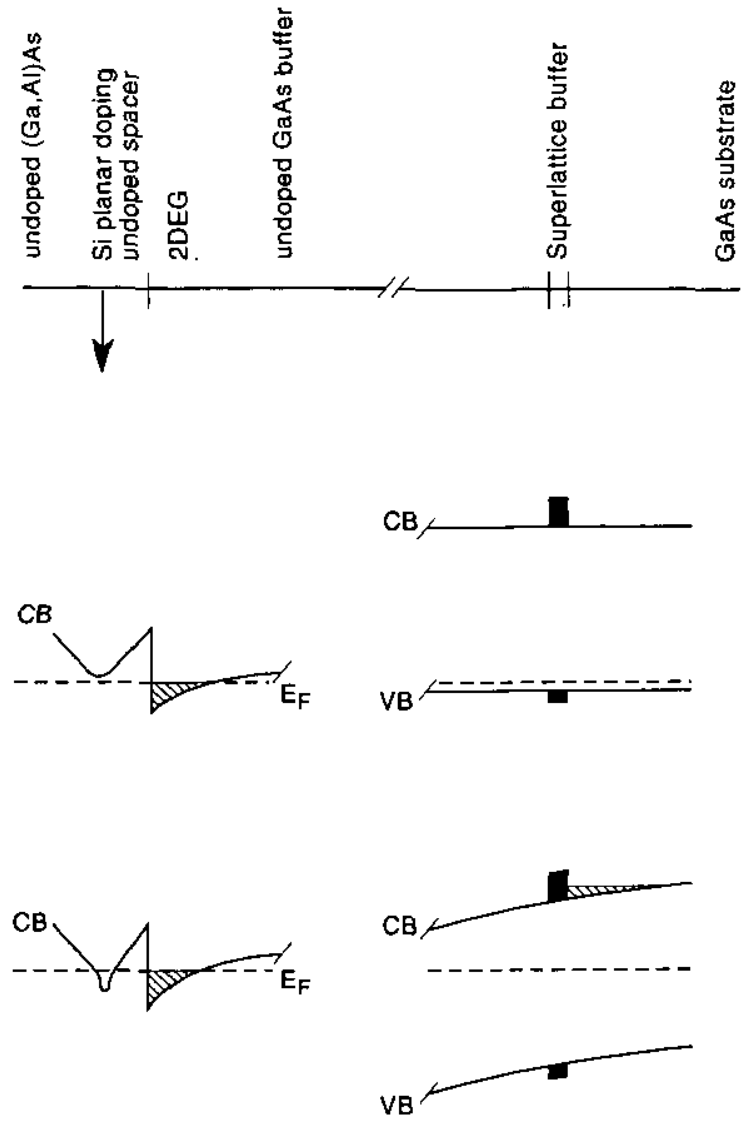

Figure 6. Growth scheme of the heterostructure (top), and spatial dependence of the conduction band potential in the growth direction: (middle) before illumination; (bottom) after saturation of the 2DEG carrier density by bursts of red light, when the depletion width has become so large that it reaches beyond the superlattice buffer.

\subsection{Carriers in the $(\mathrm{Ga}, \mathrm{Al}) \mathrm{As}$ layer}

Carriers in the $(\mathrm{Ga}, \mathrm{Al})$ As layer, which are expected to be responsible for the parallel conduction observed in magnetotransport, have a much higher effective mass value $\left(m^{*} \approx 0.088 m_{\mathrm{e}}\right.$ [16]) than electrons in a lowdensity 2DEG. Furthermore, a linewidth of a fcw teslas has been observed by Koenraad et al [17] in the FIR transmission of a $\mathrm{Si} \delta$-doping layer in GaAs. These authors attributed the broadening to the low mobility of the carriers as a result of ionized impurity scattering [18] and to the large carrier density (dielectric broadening [10]). The linewidths observed by Koenraad and coworkers strongly contrast with the linewidths we have found for the $\mathrm{T}_{3}$ resonance of $\approx 0.06 \mathrm{~T}$. Therefore the conclusion must be that it is very improbable that the $\mathrm{T}_{3}$ resonance is due to carriers in the $(\mathrm{Ga}, \mathrm{Al})$ As layer.

\subsection{Bulk GaAs carriers}

An alternative explanation of the $T_{3}$ resonance is that it could be due to 3D electrons in a GaAs layer of the samples. As the substrate is semi-insulating, the Fermi level is expected to be pinned to deep levels related to residual dopants [19], so that bulk GaAs electrons in the substrate are very unlikely to be the cause of the $T_{3}$ resonance. However, the subband oc- cupancies in these samples indicate that they possess an accumulation layer-like character (very low $N_{\mathrm{A}}-N_{\mathrm{D}}$ ) after illumination with red light $\left(\hbar \omega>E_{\mathrm{g}}\right)$ [20]. At high carrier densities it might then be possible to get a true accumulation layer with some $3 D$ electrons in the GaAs layer, between the superlattice buffer and the $(\mathrm{Ga}, \mathrm{Al}) \mathrm{As}$, leading to parallel conduction and the presence of the $\mathrm{T}_{3}$ resonance. However, the $\mathrm{CR}$ of bulk electrons in $n-G a A s$ should be of $3 D$ character, and the resonance field should therefore be completely independent of tilt angle, for all laser energies, in contradiction to the observed tilted-field behaviour. So bulk GaAs electrons are not a probable cause of the $T_{3}$ resonance.

\subsection{Impurity effects}

Fletcher et al [21] have suggested that a mobility edge may exist at the bottom of the second subband in $\mathrm{GaAs}-(\mathrm{Ga}, \mathrm{Al})$ As heterojunctions, below which a tail of localized states is found. As the $T_{3}$ resonance has some of the character of an impurity-shifted CR, we consider the possibility of $\mathrm{T}_{3}$ being associated with Fletcher's localized states. In recent publications different influences of impurities on the FIR response of 2DEG systems have been reported $[10,22-24]$. In a magnetic field the localized states would exist at the low-energy sides of the second-subband Landau levels. The energy spread of such a tail would have the correct order of magnitude to lead to a resonance of the localized states which is $\approx 0.25 \mathrm{meV}$ higher in energy than the second subband $\mathrm{CR}$ at the same field. However, as the localized states are lower in energy than the lowest Landau level of the second subband, they should already be populated at a smaller light dose than that at which second subband occupation occurs. Thus the onset of the $T_{3}$ resonance would be expected to occur at a lower light dose than the dose at which the second subband $C R$ becomes observable. As $\mathrm{CR}_{1}$ is observed before $\mathrm{T}_{3}$ in all experiments in which the light dose (and thus $N_{\mathrm{s}}$ ) has been increased, it is unlikely that impurities or localized states associated with $E_{1}$ are the origin of the $T_{3}$ resonance.

\subsection{A (metastable) secondary $2 \mathrm{DEG}$}

We suggest that the origin of the additional resonance is a (metastable) shallowly bound 2DEG in a layer behind the superlattice buffer. Such a secondary 2DEG might well be formed as a result of illumination of the sample with red light $\left(\hbar \omega>E_{\mathrm{g}}\right.$ ). The physical mechanism might be the following: before illumination of the sample the conduction band is assumed to have the structure shown in the middle diagram of figure 6 . In this case, the depletion width is smaller than the thickness of the GaAs buffer layer, so that the band is flat at the position of the GaAs-AlAs superlattice buffer. The electron-hole pairs generated by the first bursts of the red LED recombine in the regions where the conduction and valence bands are flat (among others the superlattice buffer), but are separated and thus 
prevented from recombining in the region where the conduction band has a strong slope, i.e. close to and within the depletion region. The electrons move to the 2DEG, thus causing an increase of the carrier density; the holes partly neutralize the ionized acceptors, and this leads to a decrease of the depletion charge density so that the depletion region moves towards the superlattice buffer with further illumination. These processes will cause a decrease of the electric field in the GaAs buffer close to the 2DEG and an increase of the depletion width, since the product of the two is related to the GaAs bandgap [25]. After a certain amount of illumination the depletion region will reach the superlattice buffer. The final situation is shown in the lower diagram of figure 6 , where the superlattice buffer is well within the depletion region. Due to the superlattice periodicity, minibands are formed $[26,27]$, with widths and spacings of the order of $100 \mathrm{meV}$; the bottom of the lowest miniband is about $100 \mathrm{meV}$ higher than the bottom of the GaAs conduction band [26], and this represents a considerable potential barrier for the $\mathrm{CB}$ electrons: combined with the electric field in the depletion layer this forms a secondary potential minimum behind the superlattice. Together these effects produce an accumulation of electrons at the substrate side of the superlattice, and we suggest that the $T_{3}$ resonance originates from this electron accumulation layer. Because of the very weak confinement of these electrons a quasi$3 \mathrm{D}$ character is displayed, i.e. 2D behaviour at the lower energies, and a gradual transition to $3 \mathrm{D}$ behaviour at the highest energies.

The process explained above implies that a large light dose is necessary to observe the $\mathrm{T}_{3}$ resonance. As a large light dose also leads to parallel conduction in the $(\mathrm{Ga}, \mathrm{Al})$ As layer (see section 2), the fact that the $\mathrm{T}_{3}$ resonance appears after the onset of this type of parallel conduction is coincidental.

The fact that the $T_{3}$ resonance is not observed at very low and very high laser energies is expected to be a result of the fact that it becomes indistinguishable from CR of the lowest subband, $\mathrm{CR}_{0}$.

The effective mass of the $T_{3}$ resonance is very similar, both in absolute value and in behaviour, to the cyclotron mass of 2D carriers in low-density GaAs(Ga, Al)As heterojunctions. As in low-density heterojunctions, the effective mass shows a strong increase at high FIR energies due to the resonant polaron effect [9]. The linewidths of the $T_{3}$ resonances are very similar to the values reported in $[9,10]$. Thus we conclude that the $T_{3}$ resonance behaves as the $\mathrm{CR}$ of a low-density 2DEG with a rather high mobility.

Finally, in measurements of the FIR transmission through sample 2 at $N_{\mathrm{s}}=11.25 \times 10^{15} \mathrm{~m}^{-2}$ and at $T \approx 20 \mathrm{~K}$, it appears that the $\mathrm{T}_{3}$ resonance shows a tendency to decay with time on a timescale of typically several minutes. This indicates a kind of metastability of the secondary $2 \mathrm{DEG}$ bound to the superlattice which might be due to electrons tunnelling out through the minibands of the superlattice buffer (via X-conduction band states in the barriers [29]), the process being ther- mally activated $\left(k_{\mathrm{B}} T \approx 2 \mathrm{meV}\right.$ at $\left.T=20 \mathrm{~K}\right)$.

\section{Summary and conclusions}

After a certain dose of illumination with red light $(\hbar \omega>$ $E_{\mathrm{g}}$ ) parallel conduction is observed in MBE-grown samples with planar Si doping in the $(\mathrm{Ga}, \mathrm{Al})$ As. This parallel conduction is due to a large density $\left(\approx 5 \times 10^{16} \mathrm{~m}^{-2}\right)$ of carriers with a low mobility $\left(\approx 0.08 \mathrm{~m}^{2} \mathrm{~V}^{-1} \mathrm{~s}^{-1}\right)$ after saturation of the carrier density. The origin of this parallel conduction is the $\delta$-doping layer in the $(\mathrm{Ga}, \mathrm{Al}) \mathrm{As}$.

All additional resonance, denoted as $T_{3}$, has been observed in the FIR transmission of two of our samples, after strong illumination with red light. The effective mass extracted from the position of this resonance shows an energy dependence which within experimental accuracy is similar to the energy dependence of the cyclotron mass of electrons in a low-density heterojunction. The resonance is rather narrow, with a linewidth of $\approx 0.06 \mathrm{~T}$, which indicates that the associated carriers have a long relaxation time and a low density. This completely rules out the idea that the electron sheet at the planar Si dopants, responsible for the parallel conduction seen in magnetotransport, is also the cause of the $T_{3}$ resonance. Various possible origins of the $\mathrm{T}_{3}$ resonance were discussed, and the conclusion was reached that there are at least two parallel-conducting layers in our samples: the $(\mathrm{Ga}, \mathrm{Al}) \mathrm{As}$ layer and a layer of electrons near the superlattice buffer. The electrons in this layer are expected to be weakly bound in a shallow potential well formed hy the superlattice barrier and the clectric ficld in the depletion region, and are assumed to be the origin of the $T_{3}$ resonance. The observed tiltedfield behaviour, indicating a transition from $2 \mathrm{D}$ to $3 \mathrm{D}$ character when the FIR energy increases from $7.6 \mathrm{meV}$ to $17.6 \mathrm{meV}$, and the fact that the $T_{3}$ resonance only appears after the onset of parallel conduction, support this assumption.

\section{Acknowledgments}

This work was supported by the Stichting voor Fundamenteel Onderzoek der Materie (FOM) with financial help from the Nederlandse Organisatie voor Wetenschappelijk Onderzoek (NWO). The project was also sponsored by the SCIENCE program of the European Community.

\section{References}

[1] Schubert E F, Ploog K, Dämbkes H and Heime K 1984 Appl Plys. A 3363

[2] Kirk W P, Kobiela P S, Shih H D and Reed M A 1987 High Magnetic Fields in Semiconductor Physics (Springer Series in Solid State Sciences vol 71) ed G Landwehr (Berlin: Springer) p 122

[3] Kane M J, Apsley N, Anderson D A, Taylor L L and Kerr T 1985 J. Phys. C: Solid State Phys. 185629

[4] Schubert E F, Ploog K, Dámbkes H and Heime K 1984 Appl. Phys. A 3363 
Stormer H L, Gossard A C, Wiegmann W and Baldwin K 1981 Appl. Phys. Lett. 39912

Kastalsky A and Hwang J C M 1984 Appl. Phys. Lett. 44333

[5] Maude D K, Portal J C, Dmowski L, Foster F, Eaves L, Nathan $\mathbf{M}$, Heiblum $M$, Harris $J \mathrm{~J}$ and Beall R B 1987 Phys. Rev. Lett. 59815

Chadi D J and Chang K J 1988 Plyss. Rev. Lett. 61873

Lang D V and Logan R A 1977 Phys. Rev. Lett. 39635

[6] The MBE samples were grown at the Cavendish Laboratory, Cambridge, UK: sample 1, with spacer width $5 \mathrm{~nm}$, was denoted as A227; sample 2, with spacer width $10 \mathrm{~nm}$, as A232; sample 3, with spacer width $20 \mathrm{~nm}$, as $\mathrm{A} 233$.

[7] Zrenner A, Koch F, Williams R L, Stradling R A, Ploog K and Weimann G 1988 Semicond. Sci. Technol. 31203

[8] Sigg H, Bluyssen H J A and Wyder P 1984 IEEE J. Quantum Electron. 20616

[9] Langerak C J G M, Singleton J, van der Wel P J, Perenboom J A A J, Barnes D J, Nicholas R J, Hopkins M A and Foxon C T B 1988 Phys. Rev. B 38 13133

[10] Nicholas R J, Hopkins M A, Barnes D J, Brummell M A, Sigg H, Heitmann D, Ensslin K, Harris J J, Foxon C T and Weimann G 1989 Phys. Rev. B 3910955

[11] Hopkins M A, Nicholas R J, Pfeffer P, Zawadzki W, Gauthier D, Portal J C and DiForte-Poisson M A 1987 Semicond. Sci. Technol. 2568

[12] Sigg H, Perenboom J A A J, Pfeffer P and Zawadzki W 1987 Solid State Commun. 61685

[13] Huant S, Grynberg M and Martinez G 1988 Solid State Commun. 65457

[14] Brummell M A, Hopkins M A, Nicholas R J, Portal J C, Cheng K Y and Cho A Y 1986 J. Phys. C: Solid State Phys. 19 L107
[15] Ando T 1982 J. Phys. Soc. Japan 51 3893, 3900

[16] 1982 Landolt-Bömstein, Numerical Data and Functional Relationships in Science and Technology vol 17 (Berlin: Springer)

[17] Koenraad P M, Blom F A P, Langerak C J G M, Leys M R, Perenboom J A A J, Singleton J, Spermon S J R M, van der Vleuten W C, Voncken A P J and Wolter J H 1990 Semicond. Sci. Technol. 5 861

[18] van Hall P J, Klaver T and Wolter J H 1988 Semicond. Sci. Technol. 3120

van Hall P J 1989 Superlatt. Microstruct. 6213

[19] Holmes D E, Chen R T, Elliott $\mathbf{K}$ R and Kirkpatrick C G 1982 Appl. Phys. Lett. 40 46

[20] Harris J J, Lacklison D E, Foxon C T, Selten F M, Suckling A M, Nicholas $R \mathrm{~J}$ and Barnham $\mathrm{K}$ W J 1987 Semicond. Sci. Technol. 2783

[21] Fletcher R, Zaremba E, D'Iorio M, Foxon C T and Harris J J 1988 Phys. Rev. B 387866

[22] Sigg H, Richter J, von Klitzing K and Ploog K 1989 Spectroscopy of Semiconductor Microstructures (NATO $A S I$ Series B vol 206) ed G Fasol et al (London: Plenum) p 471

[23] Ramdas A K and Rodriguez S 1981 Rep. Prog. Phys. 44 1298

[24] Richter J, Sigg H, von Klitzing K and Ploog K 1989 Phys. Rev. B 396268

[25] Bastard G 1984 Surf. Sci. 142284

[26] Maan J C 1984 Two-Dimensional Systems, Heterostructures and Superlattices (Springer Series in Solid State Sciences vol 53) ed G Bauer et al (Berlin: Springer) p 183

[27] Esaki L and Chang L L 1974 Phys. Rev. Lett. 33495

[28] Merlin R 1987 Solid State Commun. 6499

[29] Moore K J, Dawson P and Foxon C T 1988 Phys. Rev. B 383368 